Check for updates

Cite this: RSC Adv., 2018, 8, 24101

Received 13th May 2018

Accepted 26th June 2018

DOI: $10.1039 / c 8 r a 04073 g$

rsc.li/rsc-advances

\section{Discovery of acylphloroglucinol-based meroterpenoid enantiomers as KSHV inhibitors from Hypericum japonicum $\uparrow$}

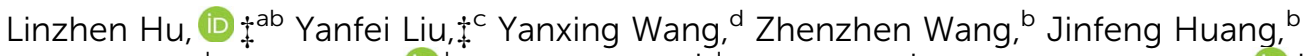 \\ Yongbo Xue, Junjun Liu, $\mathbb{D}^{\mathrm{b}}$ Zhenming Liu, ${ }^{\star d}$ Yong Chen*a and Yonghui Zhang (iD *b
}

Kaposi's sarcoma associated herpesvirus (KSHV) has gained considerable attention as a type of carcinogenic pathogen. Recent research suggests that KSHV has participated in the pathogenesis of Kaposi's sarcomarelated malignant neoplastic diseases. Viral lytic infection might be pivotal for the etiopathogenesis of KSHV-induced diseases; however, most clinical KSHV lytic replication inhibitors like ganciclovir, nelfinavir, or cidofovir do not restrain virus replication effectively enough to achieve clinical efficacy. In our continued pharmaceutical studies on Chinese herbal medicines, new acylphloroglucinol-based meroterpenoid enantiomers have been discovered from Hypericum japonicum. Most of these metabolites have potential inhibitory activities that target KSHV lytic replication. Amongst these analogues, compounds $1 \mathrm{a}$ and $1 \mathrm{~b}$ possess an unreported ring system cyclopenta[b]chromene. Compounds 1a with 4a exhibit stronger inhibitory activities towards the lytic replication of KSHV in Vero cells. In addition, $1 \mathrm{a}$ and $4 \mathrm{a}$ have $\mathrm{IC}_{50}$ values of 8.30 and $4.90 \mu \mathrm{M}$ and selectivity indexes of 23.49 and 25.70, respectively. Qualitative and quantitative SAR and molecular docking studies for acylphloroglucinol-based meroterpenoids with regard to anti-KSHV activity were conducted. An explanation for the variation in the activity and selectivity indexes was proposed in accordance with the predicted binding pose found with molecular docking to a putative target, thymidylate synthase (kTS). Compounds $1 \mathrm{a}$ and $4 \mathrm{a}$ have potential for further development and optimization of their anti-KSHV activities which could lead to new candidate drugs.

\section{Introduction}

Kaposi's sarcoma-associated herpesvirus (KSHV), containing double-stranded DNA, is in the family of Herpesviridae and within the subfamily of gamma-herpesvirus. ${ }^{1}$ It is thought to participate in the pathogenesis of Kaposi's sarcoma-related malignancies, such as multicentric Castleman's disease, AIDSrelated multicentric Castleman's disease, and primary

\footnotetext{
${ }^{a}$ National \& Local Joint Engineering Research Center of High-throughput Drug Screening Technology, Hubei Key Laboratory of Biotechnology of Chinese Traditional Medicine, School of Life Sciences, Hubei University, Wuhan 430062, Hubei Province, P. R. China. E-mail: cy101610@qq.com

${ }^{b}$ Hubei Key Laboratory of Natural Medicinal Chemistry and Resource Evaluation, School of Pharmacy, Tongji Medical College, Huazhong University of Science and Technology, Wuhan 430030, Hubei Province, P. R. China. E-mail: zhangyh@mails. tjmu.edu.cn

'The Central Hospital of Wuhan, Huazhong University of Science and Technology, Wuhan 430014, Hubei Province, P. R. China

${ }^{d}$ State Key Laboratory of Natural and Biomimetic Drugs, School of Pharmaceutical Sciences, Peking University, Beijing 100191, P. R. China. E-mail: zmliu@bjmu.edu.cn $\dagger$ Electronic supplementary information (ESI) available: 1D and 2D NMR, MS, UV, IR spectra for $\mathbf{1 - 4}$, and computational ECD data of $\mathbf{1 b}, \mathbf{2} \mathbf{a}, \mathbf{2} \mathbf{b}$, and $\mathbf{3} \mathbf{b}$. See DOI: $10.1039 / \mathrm{c} 8 \mathrm{ra} 04073 \mathrm{~g}$

$\ddagger$ These authors contributed equally to this work.
}

effusion lymphoma. ${ }^{1 a, 2}$ Like other herpesviruses, KSHV has two life cycles of latency and lytic replication, and both contribute to virus-associated disease. ${ }^{2 a}$ Generally, during the latent infection, KSHV is established as episomes via a certain amount of latent genes expressed to support viral latent replication. While in the lytic replication, viral genes, comprised of immediate early, early, and late phases, are expressed, leading to the production of mature progeny virions and ultimate lysis of cells. $^{2 b}$ Since viral lytic infection might be pivotal for the etiopathogenesis of KSHV-induced diseases, most clinical agents, including ganciclovir, nelfinavir, or cidofovir, target the inhibition of KSHV lytic replication. ${ }^{3}$ However, most of these antiviral drugs do not restrain virus replication effectively enough to achieve clinical efficacy. ${ }^{3,4}$ Therefore, additional antiviral regimens are needed which concentrate on developing candidate drugs or lead compounds which inhibit on KSHV lytic replication.

Natural products continue to arouse the interest of pharmacochemistry scientists due to their therapeutic applications and their use as an arsenal of sources for exploiting lead compounds. ${ }^{5}$ Our on-going course of systematic study on the genus Hypericum has led to an enormous amount of phloroglucinol derivatives that exhibit anti-virus activities. ${ }^{6}$ Amongst 
this genus, Hypericum japonicum Thunb (Guttiferae), a perennial or annual herb, is extensively distributed throughout North America, Oceania, and Asia. ${ }^{7}$ Extracts of the entire herb, also named "Tianjihuang" in Chinese, have been manufactured to be used by injection. Some metabolites isolated from $H$. japonicum have been demonstrated to be lead compounds with bioactivities such as anti-cancer, antimalarial, antibacterial, and anti-oxidative stress. ${ }^{8}$ With the intent of finding bioactive lead compounds from Chinese herbal medicine, our research team has investigated acylphloroglucinol-based compounds from $H$. japonicum and has discovered that most compounds exhibit potential anti-KSHV activities. ${ }^{6 c}$ Our present study of this herb-medicine led to analogue enantiomer pairs 1a/1 $\mathbf{1}-\mathbf{4 a}$ / $\mathbf{4 b}$, of which compound $\mathbf{1 a / 1 b}$ contains the novel ring system of a cyclopenta $[b]$ chromene, and compounds 1a and $\mathbf{4 a}$ exhibit stronger inhibitory activities towards the lytic replication of KSHV in Vero cells, with $\mathrm{IC}_{50}$ values of 8.30 and $4.90 \mu \mathrm{M}$, and selectivity indexes of 23.49 and 25.70 , respectively. Furthermore, the first qualitative and quantitative SAR and molecular docking studies for acylphloroglucinol-based meroterpenoids with regard to anti-KSHV activity were conducted. These studies suggest that 4a is able to anchor with kTS by hydrogen bonding to residues Arg199, Arg200, Pro217, Ser240 and Tyr282. An additional salt bridge between $5^{\prime}-\mathrm{OH}$ and Arg199 and a hydrophobic pocket formed by residues Phe115, Val158, Pro218 and other hydrophobic residues are also suggested.

\section{Results and discussion}

Enantiomeric metabolites are recognized by researchers. However, they still are infrequently studied due to the abundance of secondary metabolites. ${ }^{9}$ Often, both enantiomers have been discovered as either a scalemic or racemic mixture. ${ }^{10}$ Interestingly, another four pairs of new phloroglucinol-based terpenoid enantiomers, $( \pm)$-japonicols E-H $(\mathbf{1} \mathbf{a} / \mathbf{1} \mathbf{b}-\mathbf{4 a} / \mathbf{4 b})$, possessing diverse monoterpenoid skeletons, especially in $\mathbf{1 a} / \mathbf{1} \mathbf{b}$, with the novel carbon skeleton of a cyclopenta $[b]$ chromene, were obtained from Hypericum japonicum. The structures and absolute configurations were confirmed via extensive NMR spectroscopic data and calculated ECD analyses. Herein, the stereochemistry elucidation, biological activity measurements, as well as the Qualitative and quantitative structure-activity relationship (SAR) and molecular docking studies for these acylphloroglucinol-based meroterpenoids with regard to antiKSHV activity are presented in detail.

( \pm )-Japonicols E-H (1a/1)-4a/4b, Fig. 1) were compared (Table 1) with NMR spectroscopic data of $( \pm$ )-japonicols A-D (5a/5b-8a/8b, Fig. 1), ${ }^{6 c}$ which indicated that the japonicols E-H, the other four pairs of acylphloroglucinol-based meroterpenoid enantiomers co-occurring in the same herbs, owned the core skeleton, viz., acylphloroglucinol functional groups. These four pairs of new acylphloroglucinol-based meroterpenoid enantiomers, as racemic mixtures, were successfully isolated with almost $100 \%$ values for enantiomeric excess (ee) for each pair via separation using a CHIRALPAK ${ }^{\circ} I C$ preparative column $(10$ $\times 250 \mathrm{~mm}, 5 \mu \mathrm{m}$ particles, Daicel, China) eluted through HPLC. More information is presented in Fig. 2.

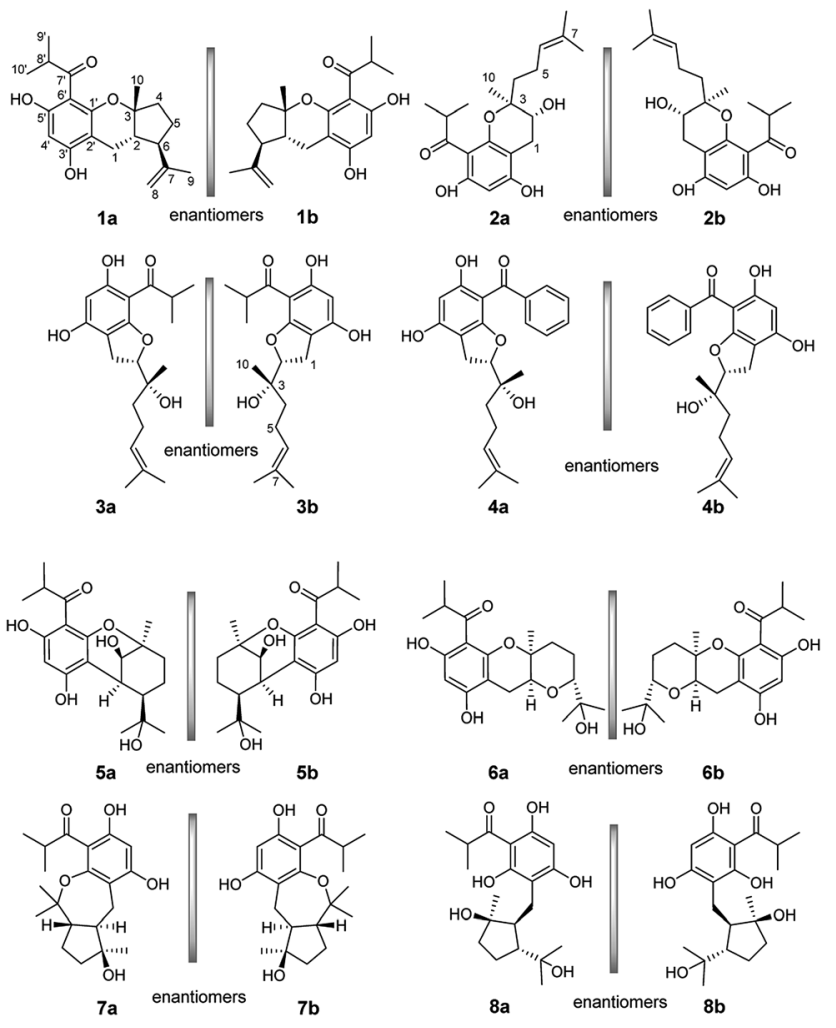

Fig. 1 Chemical structures of compounds $1 a / 1 b-8 a / 8 b$.

$( \pm)$-Japonicol E (1a/1) $)$, an off-white amorphous powder, with the molecular formula of $\mathrm{C}_{20} \mathrm{H}_{26} \mathrm{O}_{4}$, was inferred via HRESIMS data $\left(\mathrm{m} / \mathrm{z} 331.1963[\mathrm{M}+\mathrm{H}]^{+}\right.$, calcd 331.1909). The FTIR spectrogram shows that the characteristic absorptions for hydroxy $\left(3279 \mathrm{~cm}^{-1}\right)$, carbonyl $\left(1626 \mathrm{~cm}^{-1}\right)$, and aromatic ring (1593 and $1438 \mathrm{~cm}^{-1}$ ) groups on the molecule are presented. Analyses of the ${ }^{1} \mathrm{H}$ and ${ }^{13} \mathrm{C}$ NMR, including HSQC spectrum, suggested the presence of a acylphloroglucinol base along with a carbonyl $\left(\delta_{\mathrm{C}} 212.1\right)$, aromatic carbons $\left(\delta_{\mathrm{C}} 163.5,108.0,164.1\right.$, $162.2,104.8$, and one carbon resonance of $\mathrm{C}-4^{\prime}$ is not displayed in the one-dimensional NMR spectrum, but the existence of this carbon could be deduced from the HRESIMS data), two methyls $\left(\delta_{\mathrm{H}} 1.12, \mathrm{~d}, J=6.8 \mathrm{~Hz} ; 1.14, \mathrm{~d}, J=6.8 \mathrm{~Hz}\right)$, and one methine $\left(\delta_{\mathrm{H}}\right.$ $4.02, J=6.8 \mathrm{~Hz}$ ). These analyses, together with the close carbon chemical shifts of $( \pm)$-japonicols $A-D,{ }^{6 c}$ lead to the acylphloroglucinol base of the molecule. The planar structure of its monoterpene moiety was established by $\mathrm{HMBC}$ and ${ }^{1} \mathrm{H}-{ }^{1} \mathrm{H}$ COSY NMR spectra analyses (Fig. 3). The occurrence of spin systems of $\mathrm{H}-2 / \mathrm{H}-6 / \mathrm{H}-5 / \mathrm{H}-4$ in the ${ }^{1} \mathrm{H}^{-}{ }^{1} \mathrm{H}$ COSY spectrum, suggested the presence of a cyclopentane unit. Meanwhile, the long-range correlations in the $\mathrm{HMBC}$ spectrum from $\mathrm{H}_{3}-10$ to C2, C-3, and C-4, and from $\mathrm{H}_{3}-9$ to C-6, C-7, and C-8, determined the location of Me-10 and the linkage between the isoalkyl group and C-6. Ultimately, the connection of monoterpene and acylphloroglucinol motifs was demonstrated by the pivotal correlations of $\mathrm{H}-1$ with C-2, C-3, C-6, C-2' , and C-3' (HMBC), as well as $\mathrm{H}-1$ with $\mathrm{H}-2\left({ }^{1} \mathrm{H}^{-1} \mathrm{H} \mathrm{COSY}\right)$, which also established a pyran ring between the aforementioned motifs to suit the seven degrees of unsaturation. Thus, compound $\mathbf{1}$ with a ring 
Table $1{ }^{1} \mathrm{H}$ NMR $(400 \mathrm{MHz})$ and ${ }^{13} \mathrm{C}(100 \mathrm{MHz})$ data for japonicols $\mathrm{E}-\mathrm{H}(1-4)(\delta \text { in ppm, } \mathrm{J} \text { in } \mathrm{Hz})^{a}$

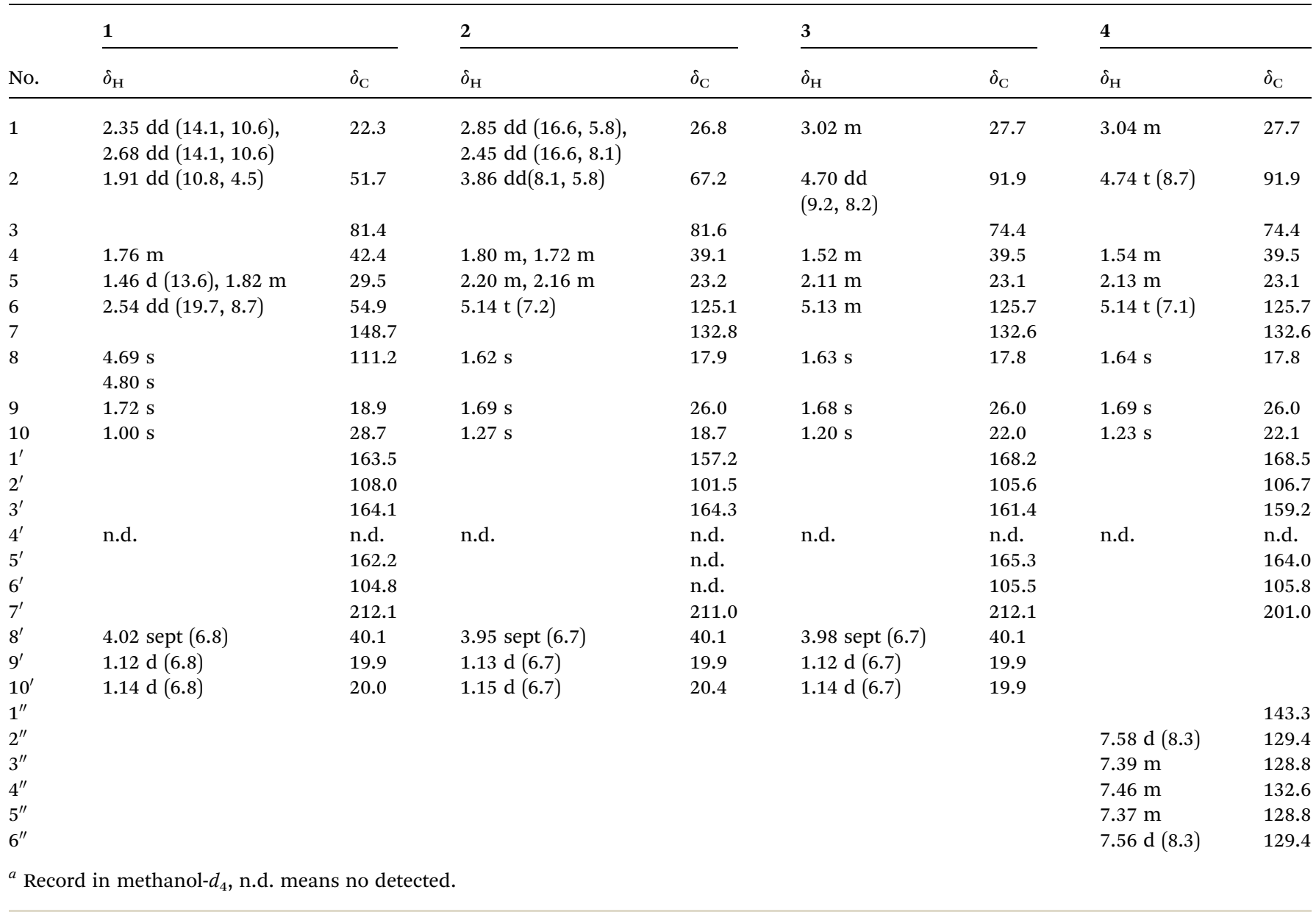
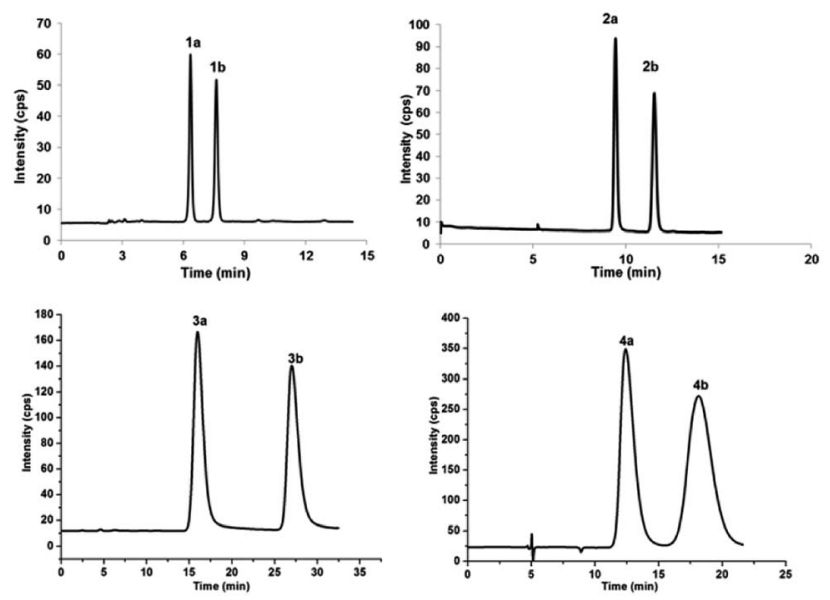

Fig. 2 Chromatograms showing the enantioseparation of racemates $1 a / 1 b-4 a / 4 b$. HPLC chromatographic specifications were the following: mobile phase of hexane/isopropanol $94 / 6(v / v)$ for $1 a / 1 b$, 99/1 (v/v) for $2 a / 2 b, 93.5 / 6.5(v / v)$ for $3 a / 3 b$, and $98 / 2(v / v)$ for $4 a / 4 b$; flow rate of $3.0 \mathrm{~mL} \mathrm{~min}{ }^{-1}$; column temperature of $25^{\circ} \mathrm{C}$; UV detection at $240 \mathrm{~nm}$; semipreparative chromatographic column, CHIRALPAK $囚 I C$ column $(10 \times 250 \mathrm{~mm}, 5 \mu \mathrm{m}$ particles, Daicel, China). All mass ratios of $1 a / 1 b-4 a / 4 b$ were roughly $1: 1$. system of a cyclopenta $[b]$ chromene was characterized, which is an unreported carbon skeleton discovered for natural product compounds.

The relative configuration was tackled by performance of NOESY correlation analyses (Fig. 3). The NOE responses of $\mathrm{H}_{3}$ $10 / \mathrm{H}-2$ assigned these two protons to the same face in an $\alpha$ orientation. Concurrently, a NOE interaction of $\mathrm{H}-6 / \mathrm{H}-8 \mathrm{~b}\left(\delta_{\mathrm{H}}\right.$ 4.80) was observed, but there was no NOE interaction between H-6 with H-2, suggesting the $\beta$-orientation of H-6 (Fig. 3).

Chiral separation of compound 1 (Fig. 2) gave rise to the pair of enantiomers $\mathbf{1 a} / \mathbf{1} \mathbf{b}$, and their absolute stereochemistry were resolved unequivocally by means of a calculated ECD spectrum method (Fig. 4) as $2 R, 3 S, 6 S$, and $2 S, 3 R, 6 R$, respectively.

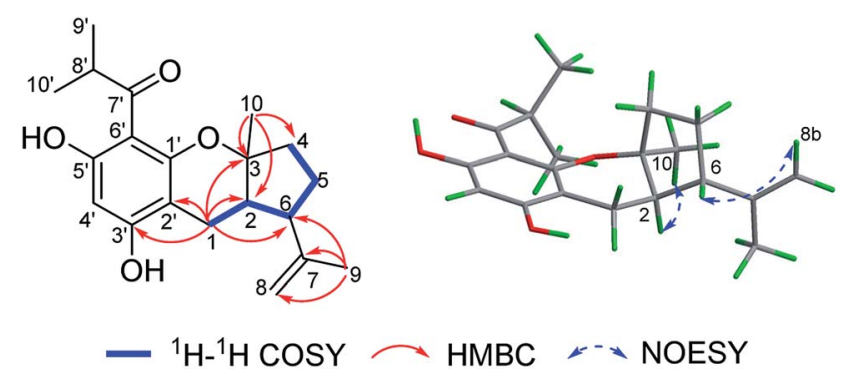

Fig. 3 Key 2D NMR correlations of compound 1. 

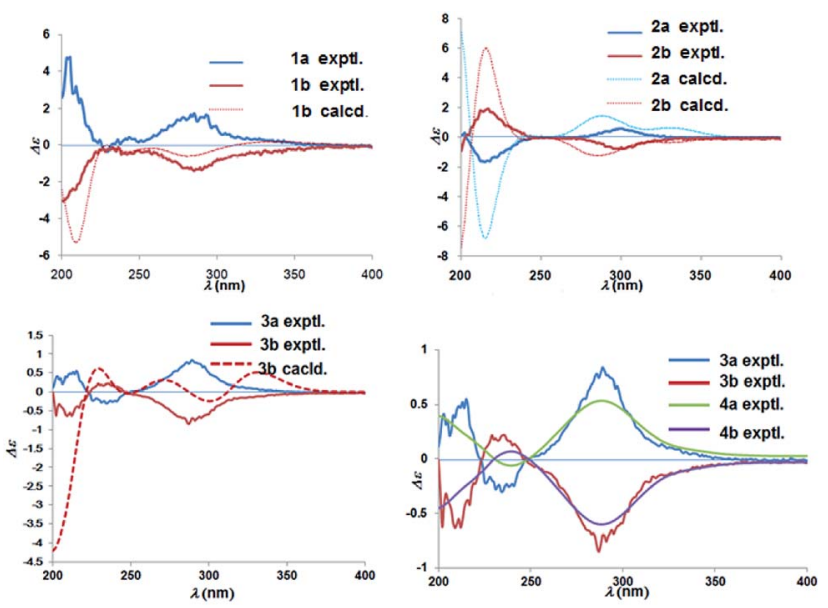

Fig. 4 Experimental ECD spectra of $1 a / 1 b-4 a / 4 b$ and calculated ECD spectra of $1 b, 2 a / 2 b$, and $3 b$.

( \pm )-Japonicol F (2a/2b), a white amorphous powder, with the molecular formula of $\mathrm{C}_{20} \mathrm{H}_{28} \mathrm{O}_{5}\left(\mathrm{~m} / z 371.1834[\mathrm{M}+\mathrm{Na}]^{+}\right.$, calcd 371.1834), was separated into two enantiomers (Fig. 2), viz. 2a and $\mathbf{2 b}$. Detailed NMR data analyses manifested that the carbon resonances of $\mathrm{C}-4^{\prime}, \mathrm{C}-5^{\prime}$, and $\mathrm{C}-6^{\prime}$ were not observed; nevertheless, the presence of these carbons was deduced from the HRESIMS data. The monoterpenoid portion along with its linkage to the acylphloroglucinol group was elucidated based on the data of $\mathrm{HMBC}$ and ${ }^{1} \mathrm{H}-{ }^{1} \mathrm{H}$ COSY correlated resonances (ESI, Fig. S1 $\dagger$ ). HMBC correlations of $\mathrm{H}_{3}-10$ with $\mathrm{C}-2 / \mathrm{C}-3 / \mathrm{C}-4$, and of $\mathrm{H} 3-8 / \mathrm{H} 3-9$ with $\mathrm{C}-6 / \mathrm{C}-7$, as well as ${ }^{1} \mathrm{H}-1 \mathrm{H}$ COSY crosspeaks of $\mathrm{H}-4 / \mathrm{H}-5 / \mathrm{H}-6$ resulted in an unambiguous assignment of the isopropenylmethyl-methyl unit, viz. C-3-C-10. The monoterpene entity is incorporated into the acylphloroglucinol unit to form a pyran ring, which was attributable to the HMBC correlations of $\mathrm{H}-1$ with $\mathrm{C}-1^{\prime} / \mathrm{C}-2^{\prime} / \mathrm{C}-2 / \mathrm{C}-3$ and the ${ }^{1} \mathrm{H}^{-1} \mathrm{H}$ COSY cross-peak of $\mathrm{H}-1 / \mathrm{H}-2$, accounting for the seven indices of hydrogen deficiency.
The relative configuration of 2 was designated through analyses of NOESY experiments. The NOE interactions of H-6 with $\mathrm{H}-5 \mathrm{~b}\left(\delta_{\mathrm{H}} 2.16\right), \mathrm{H}-5 \mathrm{~b}$ with $\mathrm{H}-4 \mathrm{~b}\left(\delta_{\mathrm{H}} 1.80\right), \mathrm{H}-4 \mathrm{~b}$ with $\mathrm{H}-1 \mathrm{~b}$ $\left(\delta_{\mathrm{H}} 2.85\right)$, and $\mathrm{H}-1 \mathrm{~b}$ with $\mathrm{H}-2$ suggested that these protons could be arbitrarily assigned as a $\beta$ orientation. On the contrary, NOE correlations of $\mathrm{H}_{3}-10$ with $\mathrm{H}-5 \mathrm{a}\left(\delta_{\mathrm{H}} 2.20\right)$ and no NOE crosspeak between $\mathrm{H}_{3}-10$ with $\mathrm{H}-2$ led to the reasonable assignment of the $\alpha$-oriented position for $\mathrm{H}_{3}-10$.

The absolute configuration of $( \pm)$-japonicol F (2a/2b), however, was eventually determined to be $2 R, 3 S$ and $2 S, 3 R$, respectively, by the well-matched theoretical ECD Cotton effects curves with the experimental spectra (Fig. 4).

$( \pm)$-Japonicol G $(\mathbf{3 a} / \mathbf{3 b})$, separated by the analogue method (Fig. 2), has the identical molecular formula of japonicol $\mathrm{F}$ (2), $\mathrm{C}_{20} \mathrm{H}_{28} \mathrm{O}_{5}$, which was affirmed by the HRESIMS with a pseudomolecular ion peak at $m / z 371.1814[\mathrm{M}+\mathrm{Na}]^{+}$(calcd 371.1834). Based on the inspection of the HMBC and ${ }^{1} \mathrm{H}^{-1} \mathrm{H}$ COSY spectra, viz, the long-range HMBC correlations of $\mathrm{H}_{3}-10$ to $\mathrm{C}-2 / \mathrm{C}-3 / \mathrm{C}-4$, $\mathrm{H} 3-8 / \mathrm{H} 3-9$ to $\mathrm{C}-6 / \mathrm{C}-7$, and $\mathrm{H}-1$ to $\mathrm{C}-2 / \mathrm{C}-3 / \mathrm{C}-1^{\prime} / \mathrm{C}-2^{\prime} / \mathrm{C}-3^{\prime}$, as well as the key COSY correlations of $\mathrm{H}-4 / \mathrm{H}-5 / \mathrm{H}-6$, and $\mathrm{H}-1 / \mathrm{H}-2$, the monoterpenoid moiety of $\mathbf{3}$ was unequivocally established as a geranyl unit, meanwhile accomplished with a furan ring to conjoin with acylphloroglucinol entity (Fig. 5A).

The side chain attached to $\mathrm{C}-2, v i z$, the geranyl group, has free rotation and the absence of one stereogenic carbon to C-2. This made it impossible to confirm the relative configurations of C-2 and C-3 merely via the NOESY signals, for instance, of harronin I and II. ${ }^{11}$ The relative configurations of the two adjacent carbons $\mathrm{C}-2$ and C-3, presented with six preferential rotamers of 3 are shown in Newman projections (Fig. 5B). The major rotamer of 3 should be I as shown in Fig. 5B, since its NOESY interactions acted in accordance with the results of NOESY experiments, viz., $\mathrm{H}-2$ with $\mathrm{H}-4 / \mathrm{H}-10$, and $\mathrm{H}-1$ with $\mathrm{H}-4$ (more details showed in ESI $\dagger$ ). Thus, the relative chiralities of C-2 and C-3 were deduced to be $2 R^{*}, 3 S^{*}$, which is consistent with the same type of stereogenic carbons in bonannione B and bonanniol D. ${ }^{12}$
A

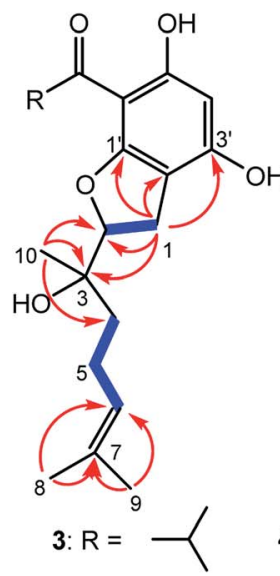

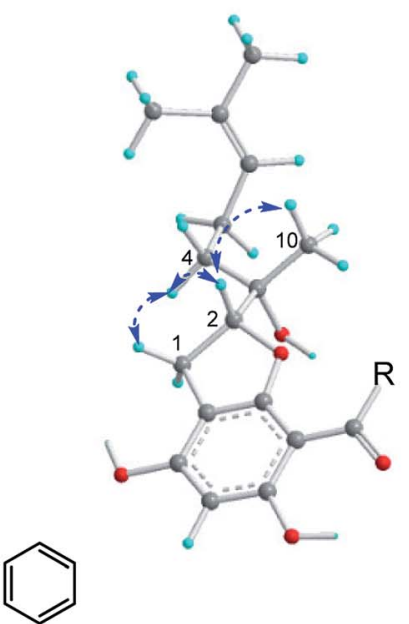

${ }^{1} \mathrm{H}-{ }^{1} \mathrm{H}$ COSY

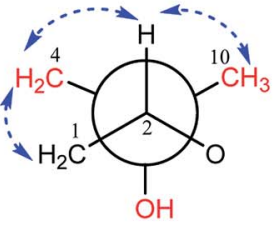

I

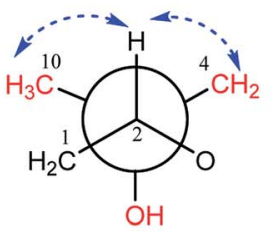

IV

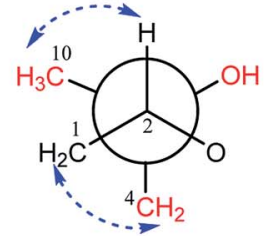

II

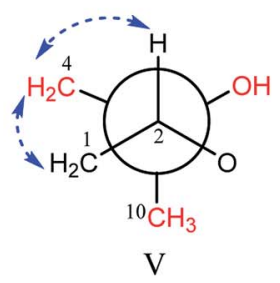

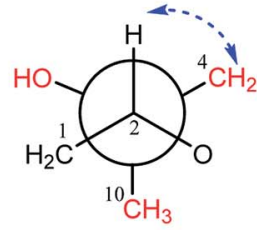

III

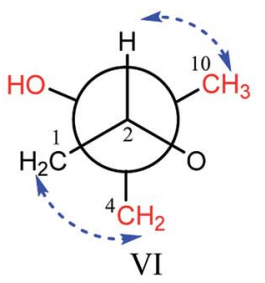

Fig. 5 (A) Key 2D NMR correlations for the core structures of 3 and 4; (B) six preferential rotamers in Newman projections for C-2/C-3 of 3. 
Table 2 Antci-KSHV activities of compounds 1a/1b-8a/8b $(\mu \mathrm{M})$

\begin{tabular}{llcl}
\hline & & & \\
Compounds & $\mathrm{CC}_{50}$ & $\mathrm{IC}_{50}$ & $\begin{array}{l}\text { Selective index } \\
\left(\mathrm{CC}_{50} / \mathrm{IC}_{50}\right)\end{array}$ \\
\hline Cidofovir $^{a}$ & $>1.00$ & 0.0081 & $>123.30$ \\
$\mathbf{1 a}$ & 195.00 & 8.30 & 23.49 \\
$\mathbf{1 b}$ & 190.20 & 24.46 & 7.78 \\
$\mathbf{2 a}$ & 126.00 & 28.00 & 4.50 \\
$\mathbf{2 b}$ & 150.20 & 27.3 & 5.50 \\
$\mathbf{3 a}$ & 79.50 & 21.73 & 3.66 \\
$\mathbf{3 b}$ & 50.10 & 6.70 & 7.48 \\
$\mathbf{4 a}$ & 125.90 & 4.90 & 25.70 \\
$\mathbf{4 b}$ & $>200.00$ & 29.46 & $>6.79$ \\
$\mathbf{5 a}$ & $>500.00$ & 202.90 & $>2.46$ \\
$\mathbf{5 b}$ & $>500.00$ & 140.90 & $>3.55$ \\
$\mathbf{6 a}$ & 140.60 & 8.75 & 16.06 \\
$\mathbf{6 b}$ & 173.70 & 29.13 & 5.96 \\
7a & 221.10 & 17.67 & 12.51 \\
7b & $>300.00$ & 39.80 & $>7.50$ \\
$\mathbf{8 a}$ & $>300.00$ & 40.00 & $>7.50$ \\
$\mathbf{8 b}$ & $>300.00$ & 158.50 & $>1.89$ \\
a & & & \\
Positive control. & & &
\end{tabular}

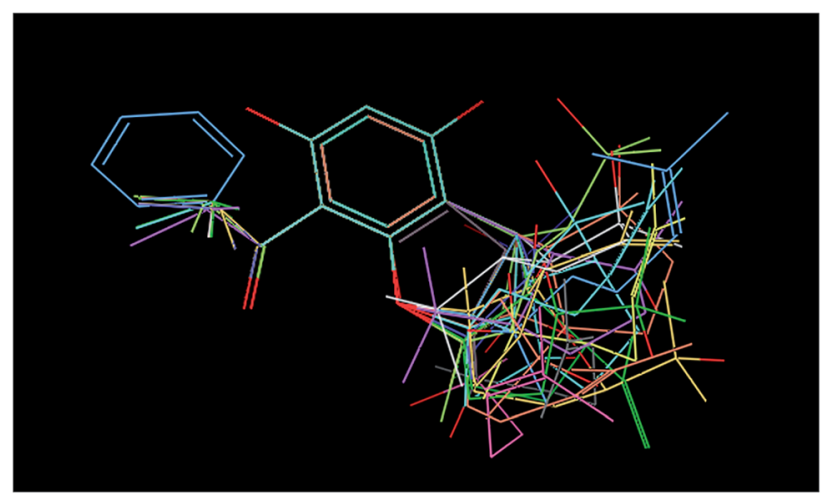

Fig. 6 Ligands were aligned through flexible shape-based alignment, which was implemented in Schrödinger Suite.
The absolute stereochemistry of C-2 was established as $2 S$ and $2 R$ for $\mathbf{3 a}$ and $\mathbf{3} \mathbf{b}$ respectively, since the ECD spectra of $\mathbf{3 a}$ and $\mathbf{3 b}$ showed similar Cotton effects with ones of peperobtusin A (negative or positive Cotton effect around $230 \mathrm{~nm}$, and positive or negative Cotton effect around $275 \mathrm{~nm}$, comparatively). ${ }^{13}$ Consequently, the absolute configurations of $( \pm$ )-japonicol $\mathrm{G}$ (3a/3b) were ascertained to be $2 S, 3 R$, and $2 R, 3 S$, respectively, which were also consistent with the matched ECD spectra between experimental and calculated ones (Fig. 4).

$( \pm)$-Japonicol $\mathrm{H}(\mathbf{4 a} / \mathbf{4 b})$ was isolated as a reddish brown oil. The only structural difference between it and japonicol $G$ was the presence of a phenyl ring instead of an isopropyl group in the latter at ${\mathrm{C}-7^{\prime}}^{\prime}$ of the acylphloroglucinol unit, which was inferred from HRESIMS data with a protonated molecular ion at $\mathrm{m} / \mathrm{z} 383.1876$ (calcd 383.1858). The enantiomeric separation procedure and $2 \mathrm{D}$ NMR correlations of $\mathbf{4}$ are presented in Fig. 2 and 5A, respectively. The relative configuration of 4 was confirmed due to its ${ }^{13} \mathrm{C}$ NMR chemical shifts at C-2 and C-3 at $\delta_{\mathrm{C}} 91.9$ and $\delta_{\mathrm{C}} 74.4$, respectively, which were identical values to those presented of compound 3 (Table 1). In comparison with $\mathbf{3 a} / \mathbf{3 b}$ (Fig. 4), the homologous ECD Cotton curves showed that $\mathbf{4 a} / \mathbf{4} \mathbf{b}$ possessed a consistent intrinsic chirality.

Biological activities of these four pairs of enantiomers $\mathbf{1 a} / \mathbf{1 b}-\mathbf{4 a}$ / 4b towards anti-KSHV activity were carried out by reported methods. $^{2 a, 6 c}$ Human iSLK.219 cells (derived from iSLK cells) inserted with rKSHV.219 [a sort of recombinant virus encoded with green fluorescent protein (GFP) and red fluorescent protein (RFP)], were utilized to assay the antiviral activity. The cytotoxicity of the compounds was measured by an AlamarBlue ${ }^{\circledR}$ Cell Viability Assay (Invitrogen), applying the protocol of the manufacturer, and was expressed as $\mathrm{CC}_{50}$ (the $50 \%$ cytotoxic concentration value). The inhibitory effects on KSHV lytic replication, assessed by infectivity assays through the GFP expression per well in Vero cells, were expressed as $\mathrm{IC}_{50}$ values (the $50 \%$ inhibiting concentration value). Effect of each compound demonstrated that 1a and 4 a possessed encouraging inhibitory effects on KSHV lytic replication in Vero cells, with $\mathrm{IC}_{50}$ values of 8.30 and $4.90 \mu \mathrm{M}$, and selectivity indexes of 23.49 and 25.70 , respectively (Table 2 ).

Table 3 Data set assignment, observed and predicted activity, and their residuals (prediction error) of training and test set

\begin{tabular}{|c|c|c|c|c|}
\hline Ligand name & QSAR set & Observed activity & Predicted activity & Prediction error \\
\hline $4 a$ & Training & 0.690 & 1.070 & -0.380 \\
\hline $4 b$ & Training & 1.469 & 1.289 & 0.180 \\
\hline $3 \mathbf{b}$ & Training & 0.826 & 1.030 & -0.204 \\
\hline $6 a$ & Training & 1.247 & 1.228 & 0.019 \\
\hline $3 \mathbf{a}$ & Training & 1.337 & 1.305 & 0.032 \\
\hline $2 \mathbf{b}$ & Training & 1.436 & 1.302 & 0.135 \\
\hline $2 a$ & Training & 1.447 & 1.319 & 0.128 \\
\hline $1 \mathbf{b}$ & Training & 1.388 & 1.384 & 0.005 \\
\hline $8 \mathbf{a}$ & Training & 1.602 & 1.426 & 0.176 \\
\hline $7 \mathbf{a}$ & Training & 0.942 & 1.355 & -0.413 \\
\hline $6 b$ & Training & 1.600 & 1.469 & 0.130 \\
\hline $5 \mathbf{b}$ & Training & 2.149 & 1.963 & 0.186 \\
\hline $8 b$ & Training & 2.200 & 2.022 & 0.178 \\
\hline $1 \mathbf{a}$ & Test & 0.919 & 1.305 & -0.385 \\
\hline $7 \mathbf{b}$ & Test & 1.464 & 1.201 & 0.263 \\
\hline $5 a$ & Test & 2.307 & 1.477 & 0.831 \\
\hline
\end{tabular}


Table 4 PLS statistics of field-based QSAR model ${ }^{a}$

\begin{tabular}{llllllll}
\hline Factors & $\mathrm{SD}_{\text {train }}$ & $R_{\text {train }}{ }^{2}$ & $F_{\text {train }}$ & $p_{\text {train }}$ & RMSE $_{\text {test }}$ & $Q_{\text {test }}^{2}$ & Pearson's $_{\text {test }}$ \\
\hline 1 & 0.2861 & 0.6184 & 17.8 & 0.00143 & 0.54 & 0.0944 \\
$2^{b}$ & 0.1922 & 0.8434 & 26.9 & $9.42 \times 10^{-5}$ & 0.55 & 0.771 \\
& & & & & & 0.7102
\end{tabular}

${ }^{a}$ Factors $=$ number of PLS factors in the model; $R^{2}=$ correlation coefficient of experimentally observed and predicted activity; SD $=$ standard deviation of regression; $F=$ variance ratio; $p=$ statistical significance; $Q^{2}=$ value of $Q^{2}$; RMSE $=$ root mean square error; Pearson's $r=$ correlation coefficient of predicted and experimentally observed activity. ${ }^{b}$ Best model.

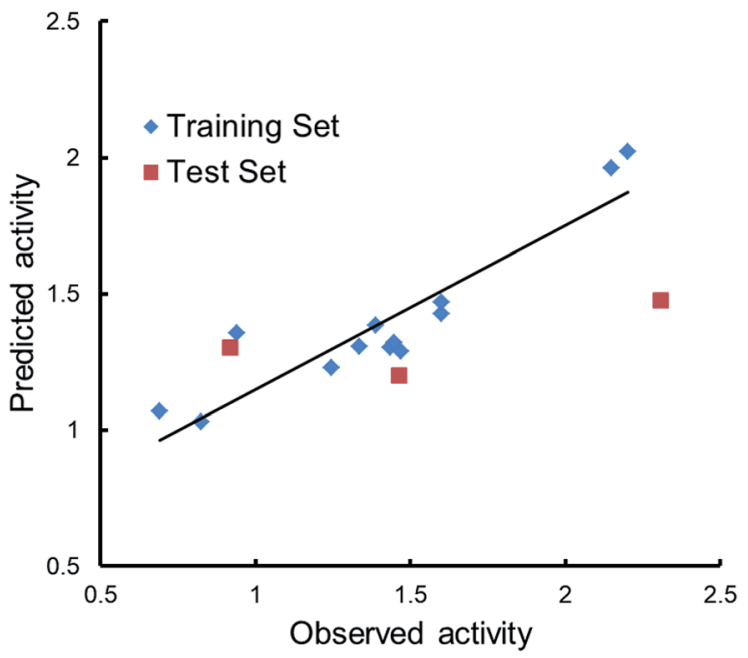

Fig. 7 Scatter plots of observed versus predicted activities.

For subsequent SAR studies of these enantiomers, the foregoing bioactivities data were used (Table 2). With a common scaffold of the acylphloroglucinol moiety, a variety of heterocycles were incorporated into the scaffold in the $1^{\prime}$ and $2^{\prime}$ positions by formation of pyran, furan, or oxepine rings. Generally, with only fused rings, addition of a remote vinyl group $(\mathbf{1 a} / \mathbf{1} \mathbf{b}-\mathbf{4 a} / \mathbf{4 b})$ caused a coarse-grained increase of $\mathrm{IC}_{50}$ when compared to $\mathbf{6 a} / \mathbf{6 b}-\mathbf{8 a} / \mathbf{8 b}$ with the vinyl group replaced by a tertiary hydroxyl group, suggesting that a polar group in that position is not tolerated. The occurrence of a bridged ring ( $5 \mathbf{a}$ / 5b) caused a decrease of the $\mathrm{IC}_{50}$, which might indicated that the flat conformation of the fused ring plays a role. Notably, given the commonness of $\mathbf{3 a}$, the enhanced activity and selectivity of $\mathbf{4 a}$ is likely attributable to the unique phenyl group at the $7^{\prime}$ position. Furthermore, it is noteworthy that the selectivity indices roughly correlate linearly with the $\mathrm{IC}_{50}$ values. However, they are entirely uncorrelated with the $\mathrm{CC}_{50}$ values.

Qualitative analysis was followed by quantitative SAR (QSAR) modelling utilizing the Field-Based QSAR Panel implemented in Schrödinger Suite ${ }^{\mathbf{1 4}}$ to set up CoMFA/CoMSIA models from these enantiomers. These enantiomers first superimposed

Table 5 Docking results for $4 a$ to $\mathrm{kPr}$ and $\mathrm{kTS}$ at SP level of precision

\begin{tabular}{ll}
\hline Receptor & Glide GScore $\left(\mathrm{kcal} \mathrm{mol}^{-1}\right)$ \\
\hline $\mathrm{kPr}(5 \mathrm{~V} 5 \mathrm{D})$ & -6.035 \\
$\mathrm{kTS}(5 \mathrm{H} 39)$ & -8.033
\end{tabular}

using the flexible shape-based alignment method (Fig. 6). The training set was used to set up the model (Table 3), and the best model (Table 4) showed moderate predictive ability. Experimental activities of additional analogues would likely provide a better model. Moreover, correlation between the observed activities and the predicted activities was plotted in Fig. 7.

To further unravel the SAR of these enantiomers, a search for all complex assemblies relevant to KSHV lytic replication in the Protein Data Bank ${ }^{\mathbf{1 5}}$ was performed, and two targets were found, namely Thymidylate Synthase (kTS) and protease (kPr). Crystal structures of kTS and kPr complexes (PDB ID: 5H39 (ref. 16) and $5 \mathrm{~V} 5 \mathrm{D},{ }^{17}$ respectively) were retrieved and $4 \mathrm{a}$ was docked into the pocket of associated reference ligands for each target using Glide. The Glide GScore of $\mathbf{4 a}$ docking to kTS was lower than
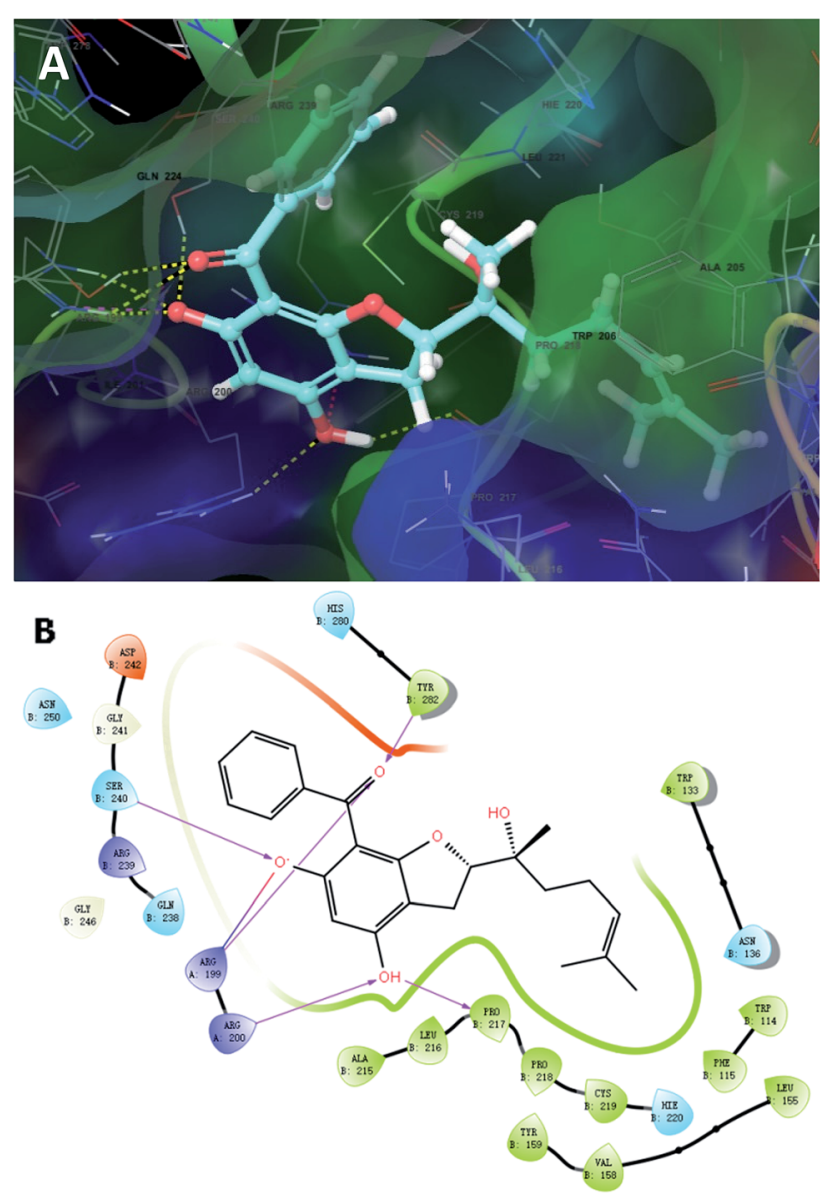

Fig. 8 Induced fit docking of $4 a$ to $k T S$ (5H39): (A) binding pose; (B) 2D interaction diagram. 
that for kPr (Table 5), and thus, kTS was assumed to be a putative target for further Induced fit docking (additionally implemented in Suite). Induced fit docking allows the receptor to alter its binding site so that it more closely conforms to the shape and binding mode of the ligand, which gave a Glide GScore of -9.326 for 4 a docking to kTS. As shown in Fig. 8, the acylphloroglucinol scaffold of 4 a was anchored via hydrogen bonding to Arg199, Arg200, Pro217, Ser240 and Tyr282 with an additional salt bridge between 5'-OH and Arg199. Moreover, the remote vinyl group was inserted in a hydrophobic pocket formed by Phe115, Val158, Pro218 and other hydrophobic residues. In general, this predictive binding mode agreed roughly with the SAR previously discussed.

\section{Conclusions}

KSHV and its viral genes are potentially associated with human malignant neoplastic diseases. Additionally, immunoassays have shown that KSHV-related antibodies remain in individuals with AIDS. ${ }^{18}$ In this research, ( \pm )-japonicols E-H (1a/1b-4a/4b) as racemic mixtures were studied. Compounds $\mathbf{1 a} / \mathbf{1} \mathbf{b}$, possess an unreported ring system of a cyclopenta $[b]$ chromene. Each pair of japonicols E-H was obtained with approximate $100 \%$ values of enantiomeric excess (ee) from Hypericum japonicum. ( \pm )-Japonicols E-H (1a/1b-4a/4b), as well as ( \pm )-japonicols A-D (5a/5b-8a/8b), were carried out the inhibition assay on KSHV lytic replication using previously developed procedures. ${ }^{6 c}$ The inhibitory effects revealed that (+)-japonicols $\mathrm{E}(\mathbf{1 a})$ and $\mathrm{H}(\mathbf{4 a})$ presented promising anti-KSHV activity in vitro along with $\mathrm{IC}_{50}$ values of 8.30 and $4.90 \mu \mathrm{M}$ and selectivity indexes of 23.49 and 25.70 , respectively. These aspects might gain insight into an extensive research for developing analogous molecules for the medical treatment of diverse KSHV associated diseases.

These enantiomers contained a variety of heterocycles incorporated into the scaffold in the $1^{\prime}$ and $2^{\prime}$ positions, leading to difficulties in analysis of their structure-activity relationships. Notwithstanding, some general correlations with the variation of $\mathrm{IC}_{50}$ and selective indices were determined. Furthermore, to find a putative target and to predict its binding mode were undertaking using molecular docking, which represents the first example of Qualitative and quantitative structure-activity relationship (SAR) and molecular docking studies for acylphloroglucinol-based meroterpenoids as antiKSHV agents. This study provides a useful starting point for further development and optimization of the anti-KSHV activities of these types of compounds and for the discovery of new therapeutic candidate drugs. Future work will include the development of synthetic routes to afford $4 \mathbf{4}$ and its analogues, and the investigation of the molecular mechanisms involved in the inhibitory effects of these types of compounds.

\section{Experimental section}

\section{General experimental procedures}

All isolated metabolites were carefully screened by analytical TLC plates (Merck, Germany) under ultraviolet-visible detector with $\lambda 254 \mathrm{~nm}$. Separations were performed by silica gel (200-
300 mesh, Yantai Chemical Co., Ltd., China), ODS (YMC Co., Japan), and Sephadex LH-20 (Mitsubishi Chemical Co., Japan) column chromatography. Generally, all target products were purified through an analytical HPLC (Shimadzu LC-10AVP Plus) with a $\mathrm{RP}^{-\mathrm{C}_{18}}$ column $\left(5 \mu \mathrm{m}, 10 \times 250 \mathrm{~mm}\right.$, Welchrom ${ }^{\circledR}$, China $)$ and a CHIRALPAK $® \mathrm{IC}$ column $(5 \mu \mathrm{m}, 10 \times 250 \mathrm{~mm}$, Daicel, China). HRESIMS data were acquired by a Thermo Scientific LTQ-Orbitrap XL apparatus. IR spectra were measured on a Bruker Vertex 70 spectrophotometer using $\mathrm{KBr}$ discs. UV spectra were recorded using a Varian Cary 50 instrument. Circular dichroism chiroptical spectra were performed using a JASCO J-1700 spectrometer. The ${ }^{1} \mathrm{H}(400 \mathrm{MHz})$ and ${ }^{13} \mathrm{C}(100$ $\mathrm{MHz}$ ) NMR spectra were obtained using a Bruker AM-600 spectrometer with tetramethylsilane (TMS) as an internal standard while the chemical shifts were characterized to solvent peaks $\left(\mathrm{CD}_{3} \mathrm{OD}, \delta_{\mathrm{H}} 3.31 \mathrm{ppm} ; \delta_{\mathrm{C}} 49.15 \mathrm{ppm}\right)$.

\section{Plant material}

The whole herbs of $H$. japonicum were harvested from the DaBie Mountain area of Hubei Province, P. R. China, in October 2011, and were authenticated by Professor Jianping Wang (School of Pharmacy, Tongji Medical College, Huazhong University of Science and Technology). A voucher specimen (ID 20111011) has been stockpiled in the Hubei Key Laboratory of Biotechnology of Chinese Traditional Medicine, Tongji Medical College, Huazhong University of Science and Technology.

\section{Extraction and isolation}

The detailed procedures of the extraction and isolation are presented in ESI. $\dagger$

( \pm )-Japonicol E (1a/1b). UV $\left(\mathrm{CH}_{3} \mathrm{OH}\right) \lambda_{\max }(\log \varepsilon) 234$ (3.53), 290 (3.68) nm; IR (KBr) $v_{\max } 3279$, 2958, 1626, 1593, 1609, $1438 \mathrm{~cm}^{-1}$; ${ }^{1} \mathrm{H}$ and ${ }^{13} \mathrm{C}$ NMR data, see Table 1 ; HRESIMS $\mathrm{m} / \mathrm{z}$ $331.1963[\mathrm{M}+\mathrm{H}]^{+}$(calcd for $\mathrm{C}_{20} \mathrm{H}_{27} \mathrm{O}_{4}, 331.1909$ ).

1 a $[(+)$-japonicol E]. White amorphous powder, $[\alpha]+82.5(c$ $\left.0.04, \mathrm{CH}_{3} \mathrm{OH}\right)$; $\mathrm{ECD}\left(\mathrm{CH}_{3} \mathrm{OH}\right) \lambda(\Delta \varepsilon) 285(+1.71) \mathrm{nm}$.

$1 \boldsymbol{b}[(-)$-japonicol E]. White amorphous powder, $[\alpha]-83.3(c$ 0.06, $\left.\mathrm{CH}_{3} \mathrm{OH}\right)$; ECD $\left(\mathrm{CH}_{3} \mathrm{OH}\right) \lambda(\Delta \varepsilon) 285(-1.40) \mathrm{nm}$.

( \pm )-Japonicol F (2a/2b). UV $\left(\mathrm{CH}_{3} \mathrm{OH}\right) \lambda_{\max }(\log \varepsilon) 292$ (4.04), 345 (3.62) nm; IR (KBr) $v_{\max } 3318,2922,2853,1618,1509$, $1421 \mathrm{~cm}^{-1}$; ${ }^{1} \mathrm{H}$ and ${ }^{13} \mathrm{C}$ NMR data, see Table 1 ; HRESIMS $\mathrm{m} / \mathrm{z}$ 371.1834 $[\mathrm{M}+\mathrm{Na}]^{+}$(calcd for $\mathrm{C}_{20} \mathrm{H}_{28} \mathrm{O}_{5} \mathrm{Na}$, 371.1834).

$2 \boldsymbol{a}[(+)$-japonicol F]. White amorphous powder, $[\alpha]+14.5(c$ 0.07, $\left.\mathrm{CH}_{3} \mathrm{OH}\right)$; ECD $\left(\mathrm{CH}_{3} \mathrm{OH}\right) \lambda(\Delta \varepsilon) 215(-1.65), 300(+0.60) \mathrm{nm}$.

$2 \boldsymbol{b}$ [(-)-japonicol $F]$. White amorphous powder, $[\alpha]-14.5(c$ 0.05, $\left.\mathrm{CH}_{3} \mathrm{OH}\right)$; ECD $\left(\mathrm{CH}_{3} \mathrm{OH}\right) \lambda(\Delta \varepsilon) 217(+1.93), 299(-0.77) \mathrm{nm}$.

( \pm )-Japonicol G (3a/3b). UV $\left(\mathrm{CH}_{3} \mathrm{OH}\right) \lambda_{\max }(\log \varepsilon) 293$ (4.32) nm; IR (KBr) $v_{\max } 3295,2970,2931,2872,1633,1609$, 1601, $1428 \mathrm{~cm}^{-1}$; ${ }^{1} \mathrm{H}$ and ${ }^{13} \mathrm{C}$ NMR data, see Table 1; HRESIMS $m / z 371.1814[\mathrm{M}+\mathrm{Na}]^{+}$(calcd for $\mathrm{C}_{20} \mathrm{H}_{28} \mathrm{O}_{5} \mathrm{Na}, 371.1834$ ).

$3 \boldsymbol{a}[(+)$-japonicol $G]$. White amorphous powder, $[\alpha]+94.2(c$ 0.05, $\left.\mathrm{CH}_{3} \mathrm{OH}\right)$; ECD $\left(\mathrm{CH}_{3} \mathrm{OH}\right) \lambda(\Delta \varepsilon) 215(+0.55), 234(-0.30), 289$ $(+0.84) \mathrm{nm}$.

3b [(-)-japonicol $G]$. White amorphous powder, $[\alpha]-93.4(c$ 0.05, $\left.\mathrm{CH}_{3} \mathrm{OH}\right)$; ECD $\left(\mathrm{CH}_{3} \mathrm{OH}\right) \lambda(\Delta \varepsilon) 209$ (-0.63), 235 (+0.22), 287 $(-0.85) \mathrm{nm}$. 
( \pm )-Japonicol $\mathbf{H}$ (4a/4b). UV $\left(\mathrm{CH}_{3} \mathrm{OH}\right) \lambda_{\max }(\log \varepsilon) 293$ (4.32) nm; IR (KBr) $v_{\max } 3243,2970,2859,1645,1566$, $1446 \mathrm{~cm}^{-1}$; ${ }^{1} \mathrm{H}$ and ${ }^{13} \mathrm{C}$ NMR data, see Table 1 ; HRESIMS $\mathrm{m} / \mathrm{z}$ $383.1876[\mathrm{M}+\mathrm{H}]^{+}$(calcd for $\mathrm{C}_{23} \mathrm{H}_{27} \mathrm{O}_{5}, 383.1858$ ).

$4 \boldsymbol{a}[(+)$-japonicol $\mathrm{H}]$. Colorless oil, $[\alpha]+87.0\left(\mathrm{c} 0.05, \mathrm{CH}_{3} \mathrm{OH}\right)$. $\operatorname{ECD}\left(\mathrm{CH}_{3} \mathrm{OH}\right) \lambda(\Delta \varepsilon) 239(-0.06), 288(+0.54) \mathrm{nm}$.

$\boldsymbol{4 b}\left[(-)\right.$-japonicol H]. Colorless oil, $[\alpha]-89.2\left(c 0.05, \mathrm{CH}_{3} \mathrm{OH}\right)$. $\operatorname{ECD}\left(\mathrm{CH}_{3} \mathrm{OH}\right) \lambda(\Delta \varepsilon) 240(+0.07), 288(-0.60) \mathrm{nm}$.

\section{Anti-KSHV assay}

Human iSLK.219 cells (derived from iSLK cells) and Vero cells were cultivated in Dulbecco's Modified Eagle's Medium (DMEM) supplemented with 10\% fetal bovine serum (Invitrogen, Carlsbad, CA, USA) in humidified incubators under $5 \%$ $\mathrm{CO}_{2}$ at $37^{\circ} \mathrm{C}$. The rKSHV.219 recombinant viruses were inserted in iSLK.219 cells with a doxycycline-inducible RTA expression system. ${ }^{19}$ The green fluorescent protein (GFP) regulated by the EF-1 $\alpha$ promoter indicated the existence of the virus, while the red fluorescent protein (RFP) regulated by the virus lytic PAN promoter manifested the occurrence of virus lytic replication. ${ }^{2 b}$

The $\mathrm{CC}_{50}$ for test compounds was executed via a previouslydeveloped procedure. ${ }^{2 b, 19}$ The iSLK.219 cells were incubated in 96-well plates ( $100 \mu \mathrm{L}$ per well). Test compounds were diluted to gradient concentration using the culture medium with doxycycline (Dox) (Beyotime) and sodium butyrate (NaB) (Sigma). The cells were then treated with the indicated compounds and cultured under $5 \% \mathrm{CO}_{2}$ at $37{ }^{\circ} \mathrm{C}$ for $48 \mathrm{~h}$. Cells without compound treatment were employed as the control. Subsequently, survival cells were assessed via AlamarBlue ${ }^{\circledR}$ Cell Viability Assay (Invitrogen). The $\mathbf{C C}_{50}$ values of the compounds were evaluated by the formula: cytoactive rate $(\%)=\left(V_{\text {treated }}-\right.$ $\left.V_{\text {blank }}\right) /\left(V_{\text {control }}-V_{\text {blank }}\right) \times 100 \%$. The values were determined by Graphpad5.0 Prism software based on the mean \pm standard deviation $(n=3)$ (ESI, Fig. S2 $\dagger$ ).

The inhibitory effects on KSHV lytic replication of the test compounds were expressed as $\mathrm{IC}_{50}$ values, which were assessed by infectivity assays according to a reported method. ${ }^{2 a}$ In brief, iSLK.219 cells were seeded in the 96-well plates until growth to $80 \%$ cell confluency. They were then treated for $48 \mathrm{~h}$ using various concentrations of compounds with the inducer (Dox and $\mathrm{NaB}$ ). Next, the supernatants of iSLK.219-treated or -untreated with the compounds were harvested after centrifugation to infect Vero cells for $48 \mathrm{~h}$. Cells without compound treatment were employed as the control, and the group without the inducer was used as the reference of the virus lytic replication. The GFP expression per well in Vero cells was analyzed through the Operetta High-Content Screening System (HCS) (Perkin Elmer), and the data of the GFP intensity per well was determined by the Harmony 3.5 software (Perkin Elmer). The $\mathrm{IC}_{50}$ values were obtained by the formula: inhibition rate $(\%)=$ $\left(V_{\text {control }}-V_{\text {treated }}\right) / V_{\text {control }} \times 100 \%$. The values were determined by mean \pm standard deviation $(n=3)$ (ESI, Fig. S2 $\dagger$ ).

\section{Computational ECD methodology}

The calculated ECD spectrum were performed for each conformer of compounds $\mathbf{1 b}, \mathbf{2} \mathbf{a} / \mathbf{2} \mathbf{b}$, and $\mathbf{3 b}$ using the TDDFT methodology at the B3LYP/6-311++G(d,p)//B3LYP/6-31G(d) level with $\mathrm{MeOH}$ as solvent by the IEFPCM solvation model implemented in Gaussian 09 program. More details are showed in ESI. $\dagger$

\section{Computational QSAR methods}

QSAR studies were conducted with Schrödinger Suite 2017-1.

\section{Field-based 3D QSAR}

A data set (Table 3) was randomly assigned into training and test sets with a ratio of approximately $4 / 1$, and their activities $\left(\mathrm{IC}_{50}\right)$ were converted to the logarithm $\left(\log \mathrm{IC}_{50}\right)$. The minimized conformations of these enantiomers were generated utilizing LigPrep and aligned according to the flexible shape-based alignment method. The force field was used and the maximum PLS factors were set as $1 / 5$ of the total number of the training set in order to avoid over-fitting. The parameters that were not mentioned were left as defaults.

\section{Molecular docking}

Binding patterns were characterized through molecular docking implemented by Glide and Induced Fit modules at the SP level of precision. The retrieved crystal structures were prepared by Protein Preparation Wizard for assigning bond orders, adding hydrogens, protonating and restraining minimizing. The best conformation of each ligand was ranked by Glide Emodel. The parameters that were not mentioned were left as defaults.

\section{Conflicts of interest}

The authors declare no competing financial interest.

\section{Acknowledgements}

This work was financially supported by the Program for Changjiang Scholars of Ministry of Education of the People's Republic of China (No. T2016088); the National natural Science Foundation for Distinguished Young Scholars (No. 81725021); Innovative Research Groups of the National Natural Science Foundation of China (81721005); the National Natural Science Foundation of China (No. 31700298, 31500281, 21502057, and 31770379); the Academic Frontier Youth Team of HUST; the Integrated Innovative Team for Major Human Diseases Program of Tongji Medical College (HUST). We thank the Analytical and Testing Center at Huazhong University of Science and Technology for assistance in testing of ECD, UV, and IR spectra. We would like to thank LetPub (www.letpub.com) for providing linguistic assistance during the preparation of this manuscript.

\section{Notes and references}

1 (a) E. Tse and Y. L. Kwong, Exp. Mol. Med., 2015, 47, e136; (b) E. De Leon Vázquez and K. M. Kaye, Arch. Virol., 2011, 156, 1323-1333. 
2 (a) J. Chen, L. Jiang, K. Lan and X. Chen, Viruses, 2015, 7, 2268-2287; (b) J. Vieira and P. M. O'Hearn, Virology, 2004, 325, 225-240.

3 S. Gantt and C. Casper, Curr. Opin. Infect. Dis., 2011, 24, 295301.

4 (a) D. P. Dittmer and S. E. Krown, Curr. Opin. Oncol., 2007, 19, 452-457; (b) H. J. Cho, S. G. Jeong, J. E. Park, J. A. Han, H. R. Kang, D. Lee and M. J. Song, Antiviral Res., 2013, 100, 75-83.

5 (a) H. Zhang, B. A. Boghigian, J. Armando and B. A. Pfeifer, Nat. Prod. Rep., 2011, 28, 125-151; (b) M. Kibble, N. Saarinen, J. Tang, K. Wennerberg, S. Makela and T. Aittokallio, Nat. Prod. Rep., 2015, 32, 1249-1266; (c) J. T. Patton, M. E. Lustberg, G. Lozanski, S. L. Garman, W. H. Towns, C. M. Drohan, A. Lehman, X. Zhang, B. Bolon, L. Pan, A. D. Kinghorn, M. R. Grever, D. M. Lucas and R. A. Baiocchi, Oncotarget, 2015, 6, 2693-2708.

6 (a) L. Hu, Y. Zhang, H. Zhu, J. Liu, H. Li, X. N. Li, W. Sun, J. Zeng, Y. Xue and Y. Zhang, Org. Lett., 2016, 18, 22722275; (b) H. Zhu, C. Chen, J. Yang, X. N. Li, J. Liu, B. Sun, S. X. Huang, D. Li, G. Yao, Z. Luo, Y. Li, J. Zhang, Y. Xue and Y. Zhang, Org. Lett., 2014, 16, 6322-6325; (c) L. Hu, Y. Xue, J. Zhang, H. Zhu, C. Chen, X. N. Li, J. Liu, Z. Wang, Y. Zhang and Y. Zhang, J. Nat. Prod., 2016, 79, 1322-1328; (d) D. Li, Y. Xue, H. Zhu, Y. Li, B. Sun, J. Liu, G. Yao, J. Zhang, G. Du and Y. Zhang, RSC Adv., 2015, 5, 5277-5287.

7 (a) Q. Pang, Y. Tian, J. Wang, J. Mi and Y. Xu, J. Pharm. Biomed. Anal., 2016, 118, 228-234; (b) L. S. Liu, M. H. Liu and J. Y. He, Molecules, 2014, 19, 10733-10754.

8 (a) S. Zhang, J. Yin, X. Li, J. Zhang, R. Yue, Y. Diao, H. Li, H. Wang, L. Shan and W. Zhang, BMC Cancer, 2014, 14, 689-699; (b) G. M. Gu, S. Z. Feng and X. Y. Wang, Acta Chim. Sin., 1988, 46, 246-251; (c) G. Y. Zuo, J. An, J. Han, Y. L. Zhang, G. C. Wang, X. Y. Hao and Z. Q. Bian, Int. J. Mol. Sci., 2012, 13, 8210-8218; (d) S. Liang, W. W. Su, Y. G. Wang, W. Peng, Y. C. Nie and P. B. Li, Int. J. Mol. Med., 2013, 32, 323-330.
9 D. J. Newman, G. M. Cragg and K. M. Snader, Nat. Prod. Rep., 2000, 17, 215-234.

10 (a) L. Wu, J. Luo, Y. Zhang, M. Zhu, X. Wang, J. Luo, M. Yang, B. Yu, H. Yao, Y. Dai, Q. Guo, Y. Chen, H. Sun and L. Kong, Tetrahedron Lett., 2015, 56, 229-232; (b) Y. Lu, Y. Xue, J. Liu, G. Yao, D. Li, B. Sun, J. Zhang, Y. Liu, C. Qi, M. Xiang, Z. Luo, G. Du and Y. Zhang, J. Nat. Prod., 2015, 78, 2205-2214.

11 R. K. Mayaka, M. K. Langat, J. O. Omolo and P. K. Cheplogoi, Planta Med., 2012, 78, 383-386.

12 (a) S. Rosselli, M. Bruno, A. Maggio, G. Bellone, C. Formisano, C. A. Mattia, S. Di Micco and G. Bifulco, Eur. J. Org. Chem., 2007, 2504-2510; (b) S. Rosselli, M. Bruno, A. Maggio, R. A. Raccuglia, M. Safder, C. Y. Lai, K. F. Bastow and K. H. Lee, Phytochemistry, 2011, 72, 942945.

13 J. M. Batista, A. N. L. Batista, D. Rinaldo, W. Vilegas, Q. B. Cass, V. S. Bolzani, M. J. Kato, S. N. López, M. Furlan and L. A. Nafie, Tetrahedron: Asymmetry, 2010, 21, 24022407.

14 Schrödinger Suite 2017-1, Schrödinger, LLC, New York, NY, 2018.

15 H. M. Berman, J. Westbrook, Z. Feng, G. Gilliland, T. N. Bhat, H. Weissig, I. N. Shindyalov and P. E. Bourne, Nucleic Acids Res., 2000, 17, 3588.

16 Y. M. Choi, H. K. Yeo, Y. W. Park and J. Y. Lee, PLoS One, 2016, 11, e0168019.

17 T. M. Acker, J. E. Gable, M. F. Bohn, P. Jaishankar, M. C. Thompson, J. S. Fraser, A. R. Renslo and C. S. Craik, J. Am. Chem. Soc., 2017, 139, 11650-11653.

18 (a) S. J. Gao, L. Kingsley, M. Li, W. Zheng, C. Parravicini, J. Ziegler, R. Newton, C. R. Rinaldo, A. Saah, J. Phair, R. Detels, Y. Chang and P. S. Moore, Nat. Med., 1996, 2, 925-928; (b) E. Cesarman, Y. Chang, P. S. Moore, J. W. Said and D. M. Knowles, N. Engl. J. Med., 1995, 332, 1186-1191.

19 J. Myoung and D. Ganem, J. Virol. Methods, 2011, 174, 12-21. 